\title{
Fibroma cemento-osificante
}

\author{
Cemento-ossifying fibroma
}

\author{
Alfio Secchi Álvarez,* Joaquín Vázquez de Ponson Du Terrail,* \\ Cristóbal Sepúlveda Verdugo,* Cristián Núñez Baeza, \\ Riveros, ${ }^{\star,}$ Alexis Carrasco Raimondi, Araceli Raposo Castillo ${ }^{\ddagger, \&}$
}

\section{RESUMEN}

El fibroma cemento-osificante (FCO) es una neoplasia benigna odontogénica, de tejido mesenquimal sin epitelio odontogénico; su localización más frecuente es a nivel de premolares y molares mandibulares, presenta mayor prevalencia en mujeres entre la segunda y cuarta décadas de la vida. En el siguiente artículo se presenta un caso de FCO, en donde se describen características clínicas, imagenológicas, histopatológicas y de tratamiento, comparándolas con la evidencia actual. El caso pertenece a una paciente atendida en el Complejo Asistencial Barros Luco, distinguiendo características epidemiológicas similares con las propuestas por la evidencia global. El tratamiento consistió en resección marginal y reconstrucción con injerto autólogo no vascularizado complementado con sticky bone y plasma rico en fibrina. El diagnóstico de FCO siempre debe ser apoyado por análisis histopatológico, complementándolo con estudios imagenológicos tridimensionales para determinar extensión y estructuras óseas o dentales comprometidas, es necesario descartar una amplia variedad de diagnósticos diferenciales que poseen presentación similar, pero comportamientos distintos. El tratamiento de esta lesión debe ser bien analizado, considerando la extensión y relación con estructuras

\section{ABSTRACT}

The cemento-ossifying fibroma (COF) is a benign odontogenic neoplasm, of mesenchymal tissue without odontogenic epithelium; its most frequent location at the level of premolars and mandibular molars, presented higher prevalence in women between the second and fourth decade. The following article presents a case of $\mathrm{COF}$, in which clinical, imaging, histopathological, and treatment characteristics are described, comparing them with current evidence. The case belongs to a patient treated at the Barros Luco Care Center; distinguishing similar epidemiological characteristics with those proposed by global evidence. The treatment consisted of marginal resection and reconstruction with nonvascularized autologous graft supplemented with sticky bone and platelet-rich in fibrin. The diagnosis of COF should always be supported by histopathological analysis, complementing it with three-dimensional imaging studies to determine the extent and compromised bone or dental structures, it is necessary to discard a wide variety of differential diagnostics that have similar presentation but different behaviors. The treatment of this lesion should be well analyzed, considering the extent and relationship with neighboring structures, the quality of life can be

\footnotetext{
* Cirujano Dentista, pasante del Servicio de Cirugía y Traumatología Maxilofacial, Complejo Asistencial Barros Luco. Chile.

₹ Cirujano Dentista, Cirujano Oral y Maxilofacial, equipo del Servicio de Cirugía y Traumatología Maxilofacial, Complejo Asistencial Barros Luco. Chile. $\S$ Docente, Departamento de Cirugía, Universidad de Chile. Chile.

\author{
Correspondencia: \\ Alfio Secchi Álvarez \\ E-mail: alfio.secchi@gmail.com
}

Citar como: Secchi ÁA, Vázquez de Ponson DTJ, Sepúlveda VC, Núñez BC, Quezada RG, Carrasco RA et al. Fibroma cemento-osificante. Rev Mex Cir Bucal Maxilofac. 2021;17 (1): 17-23. https://dx.doi.org/10.35366/100256 
vecinas, la calidad de vida puede verse afectada por el potencial aumento de tamaño y compromiso funcional, considerando que posee baja recidiva.

Palabras clave: Fibroma cemento-osificante, resección, mandíbula. affected by the potential increase in size and functional commitment, considering that it has a low recurrence.

Keywords: Cemento-ossifying fibroma, resection, mandible.

Servicio de Especialidades Odontológicas del Complejo Asistencial Barros Luco (SEO) por hallazgo radiográfico en ortopantomografía evaluada en el Centro de Salud Familiar de su comuna. Relata presentar ocasionalmente disestesia de labio inferior de más de un año de evolución. Al examen extraoral, presenta leve asimetría en zona del ángulo mandibular derecho, intraoralmente se aprecia mucosa y encías de aspecto normal, aumento de volumen en zona retromolar derecha de consistencia pétrea y asintomático a la palpación (Figura 1).

En los exámenes imagenológicos, la ortopantomografía describe diente 4.8 semi-incluido en angulación vertical, con lesión de densidad mixta distal a diente 4.8 , de $4 \times 3$ centímetros de extensión, bordes escleróticos y centro radiopaco, sobreproyectado en conducto alveolar inferior. Se solicitó tomografía computarizada en la cual se observó área de densidad mixta, circunscrita de límites definidos, distal a pieza 4.8 semi incluida y anterior a rama mandibular, evidente expansión cortical vestibular y lingual (Figura 2). Se realizó biopsia incisional en pabellón central bajo anestesia general, extrayendo muestra de tejido de consistencia dura y en conjunto se realizó exodoncia de diente 4.8 por proximidad y compromiso con lesión; el acondicionamiento y síntesis de tejidos blandos fue minucioso utilizando plasma rico en fibrina (PRF) para favorecer cicatrización. El estudio histopatológico describe lesión con proliferación de tejido fibroso, presencia de fibroblastos entremezclados en tejido mineralizado tipo cemento, vascularización moderada y ausencia de atipias celulares.

Se realizó control de cicatrización por seis semanas para garantizar ausencia de comunicación entre tejido óseo y mucosas de cavidad bucal, manteniendo una buena cobertura para la cirugía de resección con uso de injerto y osteosíntesis.

En un segundo tiempo quirúrgico, en pabellón central bajo anestesia general mediante cervicotomía derecha, se realiza resección marginal de mandíbula (completa de lesión, sin comprometer borde basilar)

Mujer de 34 años, con antecedentes de hipoti-
roidismo en tratamiento, es referida desde el 
junto a curetaje; reconstrucción inmediata con injerto autólogo de cresta iliaca derecha, uso de PRF con sticky bone como coadyuvante a la cobertura de osteosíntesis e injerto. Informe histopatológico confirma FCO como diagnóstico (Figuras 3 y 4).

En el postoperatorio el paciente presenta evolución favorable, buenas condiciones generales, presencia de paresia leve en labio inferior y dolor moderado en la zona dadora. Se deriva a kinesiólogo para iniciar fisioterapia de drenaje inflamatorio y estimulación para recuperar función completa de movilidad de labio inferior.

En dos meses postoperatorio el paciente presenta evolución favorable a terapias kinesiológicas, tejidos blandos intraorales sin alteraciones, ausencia de dehiscencias y se mantiene limitación en marcha por injerto de cresta iliaca.

\section{DISCUSIÓN}

La clasificación del FCO ha variado a lo largo del tiempo, en su primera descripción en el año 1989 fue incluido dentro de lesiones fibro-óseas no odontogénicas, considerando las similitudes histológicas en donde existe reemplazo de tejido óseo esponjoso por fibroso, incremento de fibroblastos y colágeno. ${ }^{7}$ En 1992, la World Health Organization (WHO) separó las entidades fibroma osificante y fibroma cementante de manera independiente, pero manteniendo al FCO como lesión fibro-ósea; ${ }^{1}$ a partir del 2017 la WHO actualizó los tumores odontogénicos (propios de maxilares) incluyendo al FCO como una entidad benigna de origen ectomesenquimal sin epitelio odontogénico.

Su comportamiento de lesión benigna presenta reportes de mayor agresividad en lesiones cercanas a la línea media, ${ }^{5,8}$ así como también en la presencia de mayor cantidad de cemento, ${ }^{9}$ para esta última descripción es necesario mayor evidencia que lo confirme. Bajo este escenario existen reportes de una variante juvenil agresiva en menores de 15 años, de crecimiento rápido, con mayor frecuencia en maxilar y sin predilección por sexo, denominada FCO juvenil. Su tratamiento implica resección completa de lesión con curetaje, presentando porcentaje de recidiva entre $30-60 \% .5,6,9$ Dentro de la variante juvenil (FCO juvenil) existe una subvariante, juvenil psamomatoide, de mayor frecuencia entre 16-33 años, predilección en seno maxilar y sin variación por sexo. ${ }^{10,11}$

EI FCO se presenta con mayor frecuencia entre la segunda y cuarta décadas de la vida, en sexo feme- nino con una razón 1:5 (M:F), su localización más frecuente es la mandíbula (75\% aproximadamente) en relación con la zona de molares y premolares, lo que concuerda con el caso presentado; existen también reportes en cavidad nasal y sinusal, pero son catalogados como inusuales. ${ }^{1,6}$

La evidencia describe un crecimiento lento, progresivo y generalmente asintomático, lo cual se relaciona con subdiagnósticos y/o hallazgos radiográficos. El aumento de volumen que se le asocia es localizado, de consistencia dura y podría causar asimetría facial. Estos signos son cardinales para su sospecha clínica, la expansión de estructuras blandas y duras no considera daño aparente o linfoadenopatías asociadas. A nivel intraoral la mucosa de recubrimiento rara vez posee alteraciones y en ocasiones se reporta desplazamiento de piezas vecinas y daño en raíces o rizalisis. ${ }^{6,7}$ En el caso presentado, la expansión cortical vestibular y lingual era evidente, aunque no existía rizalisis.

Su etiopatogenia continúa siendo controversial, asociándola con eventos traumáticos y procesos inflamatorios, ${ }^{1,5,12}$ el presente caso no recuerda haber sufrido traumatismo considerable en la zona, tampoco se realizaron exodoncias, pero sí existía un diente semiincluido en angulación vertical y vestibuloversión en relación íntima al diente vecino y la lesión.

A nivel imagenológico, el FCO presenta variaciones en su densidad dependiendo del estado de maduración y calcificación, ${ }^{3,12}$ en el caso descrito, la lesión se presentó bien definida, con áreas de densidad mixta en la TC y radiopaca-radiolúcida en la radiografía.

Con base en la evidencia, el comportamiento maligno del FCO es concluyente y se considera inusual o de bajo riesgo.

El diagnóstico del FCO debe ser con apoyo en exámenes de imagenología e histopatología. Diversos diagnósticos diferenciales deben ser considerados, entre ellos: displasia fibrosa, FCO juvenil, displasia cemento-ósea focal, osteoma osteoide, osteoblastoma, cementoblastoma y fibroma ameloblástico (Tabla 1).3,5

El tratamiento del FCO puede depender directamente del tamaño y del caso clínico en particular; en lesiones consideradas pequeñas y donde no existe desplazamiento de corticales o estructuras, se puede realizar enucleación y curetaje, considerando que esta técnica presenta un $28 \%$ de recidiva a los cinco años. ${ }^{2,9,13}$ La resección quirúrgica y curetaje no han presentado recidiva en seguimientos de hasta 10 años; sin embargo, es necesario planificar minuciosamente este procedimiento debido al compromiso 

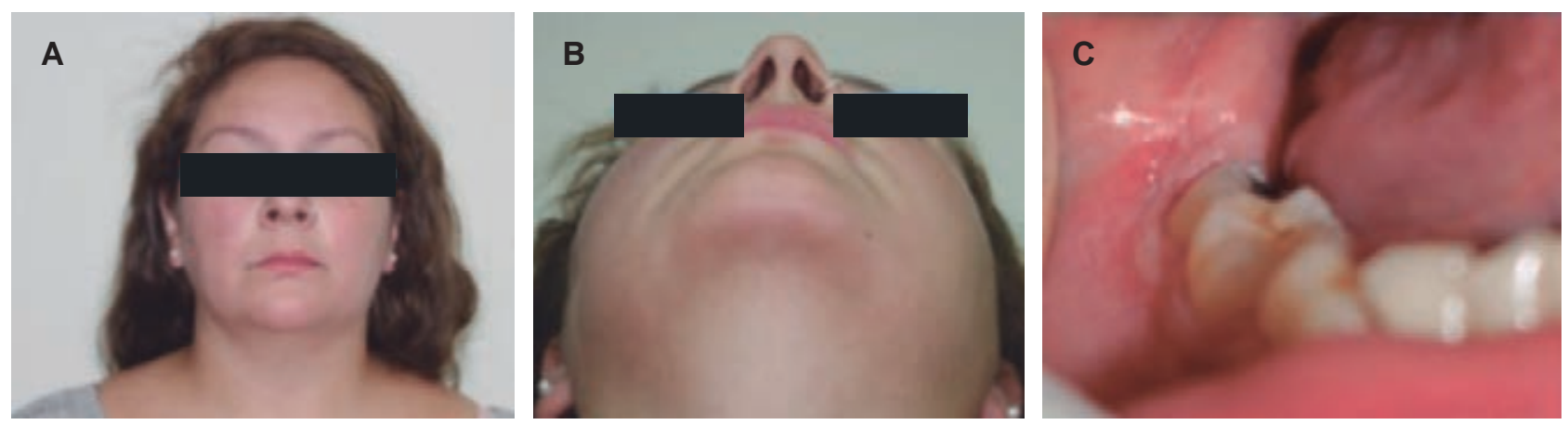

Figura 1: Examen extraoral e intraoral. A) Leve asimetría en ángulo mandibular derecho. B) Leve asimetría en contorno de borde basilar, a nivel de ángulo mandibular derecho. C) Trígono retromolar derecho, mucosa sin alteraciones.
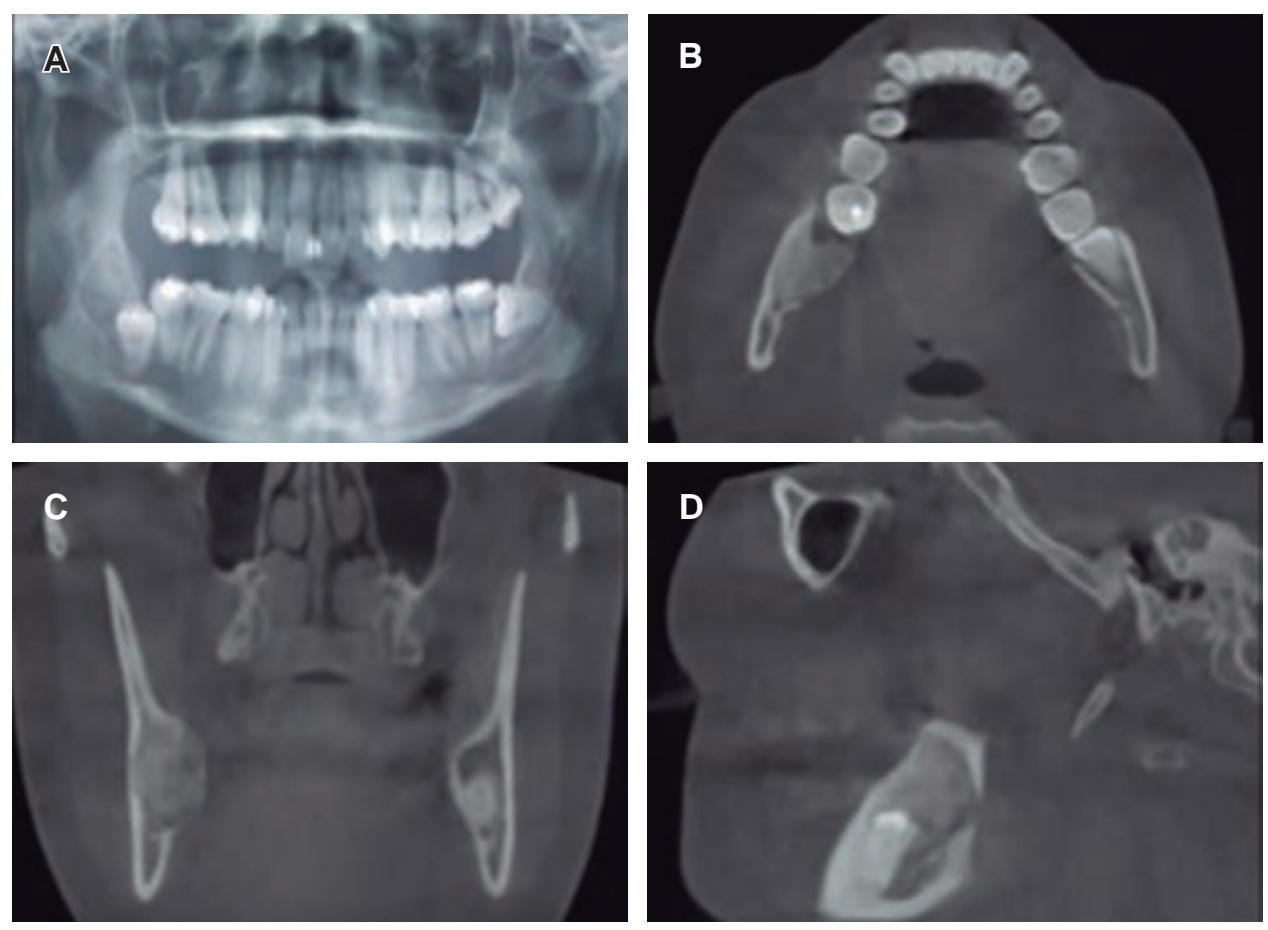

Figura 2:

Exámenes imagenológicos.

A) Ortopantomografía, lesión de densidad mixta (radiopaco y radiolúcido), bordes definidos, distal a diente 4.8 y sobreproyectado en canal mandibular. B) Tomografía computarizada (TC) corte axial: lesión densidad mixta, ángulo mandibular derecho, expansión corticales vestibular y lingual. C) TC coronal: lesión densidad mixta, ángulo mandibular derecho, expansión y erosión cortical lingual. D) TC sagital: lesión densidad mixta, ángulo mandibular derecho, en relación a diente 4.8 incluido, sin alteración en conducto alveolar inferior. de estructuras funcionales, considerando reconstrucciones con injertos autólogos no vascularizados como cresta iliaca, o vascularizados como fíbula dependiendo del tamaño y volumen de la resección. , $^{2,3}$

\section{STICKY BONE Y FIBRINA RICA EN PLAQUETAS (PRF)}

El uso de fibrina rica en plaquetas (PRF, por sus siglas en inglés) como complemento quirúrgico o terapia coadyuvante ha demostrado un potencial altamente exitoso en el territorio maxilofacial. Avances tecnológicos en el campo de la flebotomía y centrifugación han construido variaciones en el método de confección del PRF, entregando gran versatilidad en sus usos..$^{14} \mathrm{La}$ forma de obtención de matrices de fibrina autóloga se ha dirigido a un acceso universal y de menor costo, el cual presenta mayores ventajas fisiológicas medibles en mayores concentraciones de factores de crecimiento y obtención de plaquetas en contraste con la técnica de plasma rico en plaquetas (PRP). ${ }^{15,16}$

La célula plaquetaria se centra como la protagonista y posee un rol activo debido al contenido de sus gránulos que presentan variados factores de crecimiento, entre ellos, factor de crecimiento 

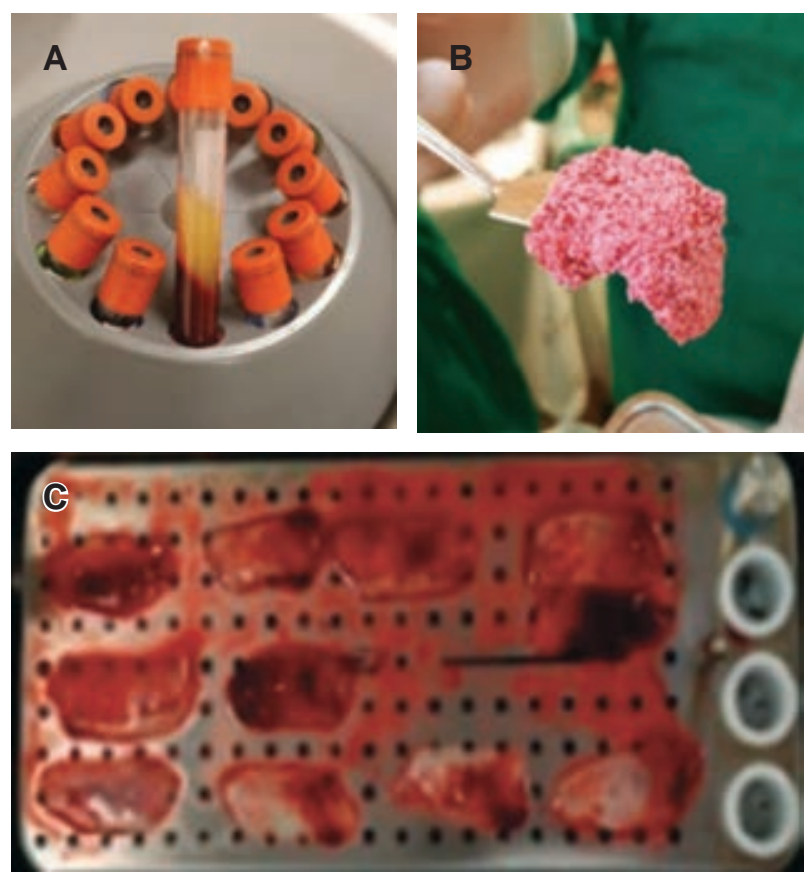

Figura 3: Proceso de plasma rico en fibrina (PRF), membranas y sticky bone. A) Procedimiento de centrifugación y preparado para PRF. B) Sticky bone preparado con particulado de hueso autólogo y membranas de PRF. C) Preparación de membranas de PRF en caja de manipulación. derivados de plaquetas (PDGF), factor de crecimiento transformante- $\beta$ (TGF- $\beta$ ), factor de crecimiento endotelial (VEGF), factor de crecimiento tipo insulina-1 (IGF-1), factor de crecimiento epidérmico (EGF), los cuales contribuyen a la cicatrización normal y acelerada de la zona intervenida. ${ }^{17}$

El proceso de centrifugación, en sus variaciones en tiempo y revoluciones, favorece la conformación de un tapón en donde se agrupa fibrina y concentrados de factores de crecimiento, los cuales por su consistencia han de ser usados como relleno para el cierre de defectos de continuidad, ser comprimidos (por medio de cajas metálicas específicas para manipulación de PRF) para la conformación de membranas de mayor densidad, para favorecer relleno estructural o ser mezclado junto con injertos óseos particulados autólogos o sintéticos para la formación de sticky bone, como fue realizado en este caso.

En este estudio, el hueso autólogo injertado junto con el particulado que conforma el sticky bone cumplen función de osteoconductor (al rellenar el espacio resecado) y osteoinductor, debido a las propiedades de las células y factores de crecimiento atrapados en los coágulos de fibrina, aportando una terapia coadyuvante y complementaria efectiva a cirugías invasivas. ${ }^{18,19}$
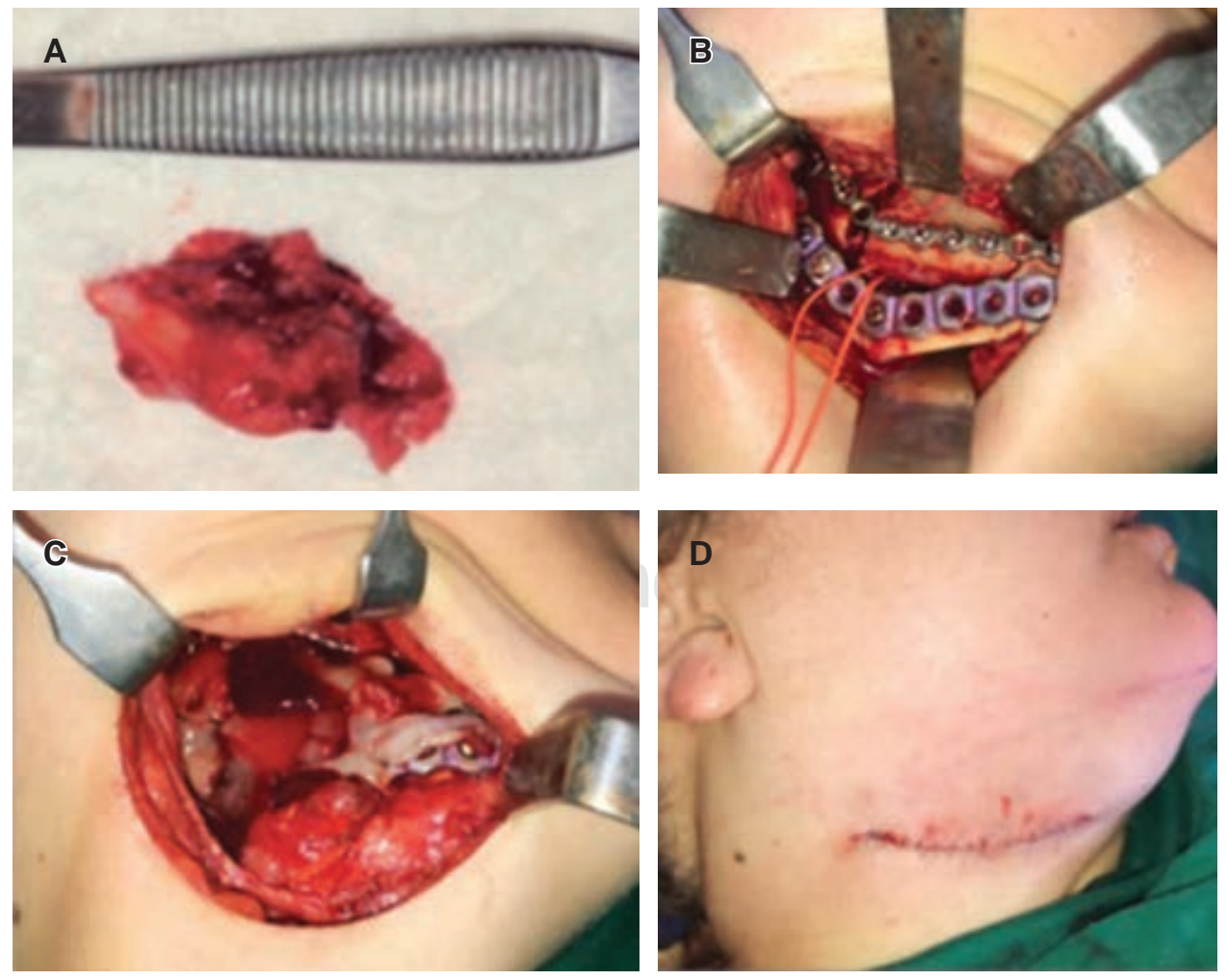

Figura 4:

Imágenes perioperatorias. A) Resección de lesión 4 $\times 3 \mathrm{~cm}$. B) Cervicotomía y placa osteosíntesis de reconstrucción en borde basilar, placa osteosíntesis e injerto de cresta iliaca. C) Aplicación de plasma rico en fibrina en membranas y sticky bone. D) Cierre y síntesis de tejidos. 
Tabla 1: Características de fibroma cemento-osificante y diagnósticos diferenciales.

\begin{tabular}{|c|c|c|c|}
\hline Lesión tumoral & Imagenología & Histopatología & Epidemiología \\
\hline $\begin{array}{l}\text { Fibroma } \\
\text { cemento-osificante }\end{array}$ & $\begin{array}{l}\text { Bien delimitado, borde esclerótico } \\
\text { y halo radiolúcido } \\
\text { Mixto, áreas radiopacas en fondo } \\
\text { radiolúcido } \\
\text { Expansión cortical, } \\
\text { desplazamiento dental, } \\
\text { rizalisis }\end{array}$ & $\begin{array}{l}\text { Estroma de tejido conectivo fibroso } \\
\text { con canales vasculares y tejido } \\
\text { mineralizado }{ }^{4,10}\end{array}$ & $\begin{array}{l}\text { Sexo femenino segunda-cuarta } \\
\text { década } \\
\text { Mandíbula posterior }{ }^{3}\end{array}$ \\
\hline $\begin{array}{l}\text { Fibroma osificante } \\
\text { juvenil }\end{array}$ & $\begin{array}{l}\text { Bien delimitada, sin presencia de } \\
\text { línea radiolúcida periférica } \\
\text { Mixto, áreas radiopacas en fondo } \\
\text { radiolúcido } \\
\text { Perforación cortical, invasión de } \\
\text { estructuras vecinas } \\
\text { 10,11,20 }^{-1}\end{array}$ & $\begin{array}{l}\text { Tejido fibroso con alta con } \\
\text { alta celularidad depósitos tipo } \\
\text { osteoide, hueso trabecular } \\
\text { rodeado por osteoclastos con } \\
\text { aumento de tamaño }\end{array}$ & $\begin{array}{l}\text { Sin predilección por sexo } \\
\text { Leve frecuencia en sexo } \\
\text { masculino } \\
\text { Maxilar asociado con senos } \\
\text { maxilares, esfenoidales }{ }^{10,11,20}\end{array}$ \\
\hline Displasia fibrosa & $\begin{array}{l}\text { Mal delimitado, sin bordes } \\
\text { Mixto, radiolúcido-radiopaco } \\
\text { (según estadio) patrón piel de } \\
\text { naranja, motas de algodón } \\
\text { Sin delimitación con cortical, } \\
\text { expansión de corticales bucal- } \\
\text { lingual; esclerosis en huesos de } \\
\text { base de cráneo, desplazamiento } \\
\text { dental }{ }^{10,14,15}\end{array}$ & $\begin{array}{l}\text { Sólo si la imagen no clarifica } \\
\text { diagnóstico } \\
\text { Estroma fibroso con fibroblastos } \\
\text { fusiforme y alta vascularidad } \\
\text { Trabeculado irregular similar a } \\
\text { letras chinas } \\
\text { Anillo periférico de osteoblastos } \\
\text { ausente }^{10,14,15}\end{array}$ & $\begin{array}{l}\text { Monostóticas: segunda-tercera } \\
\text { década (más común en } \\
\text { maxilares) } \\
\text { Maxilar posterior (más de un } \\
\text { hueso craneofacial) } \\
\text { Mandíbula con menor } \\
\text { porcentaje }{ }^{10,14,15}\end{array}$ \\
\hline $\begin{array}{l}\text { Displasia cemento ósea } \\
\text { focal }\end{array}$ & $\begin{array}{l}\text { Bien delimitada con o sin borde } \\
\text { esclerótico } \\
\text { Radiolúcido, mixta o radiopaca } \\
\text { Relación con ápice dental, tamaño } \\
\text { reducido, no agresivo }{ }^{10}\end{array}$ & $\begin{array}{l}\text { No recomendada por baja } \\
\text { vascularización de tejido óseo y } \\
\text { similitudes con otras patologías }{ }^{15,16}\end{array}$ & $\begin{array}{l}\text { Sexo femenino } \\
\text { Cuarta década } \\
\text { Primer premolar-segundo } \\
\text { molar inferior } \\
\text { Raza oscura }^{15,16}\end{array}$ \\
\hline Fibroma ameloblástico & $\begin{array}{l}\text { Bien delimitado, borde esclerótico } \\
\text { Radiolúcido uni-multilocular } \\
\text { Expansión de corticales, puede } \\
\text { estar asociado con diente en } \\
\text { erupción }^{17,18}\end{array}$ & $\begin{array}{l}\text { Imprescindible por alto porcentaje } \\
\text { de recidiva y tendencia a } \\
\text { malignidad (fibrosarcoma) } \\
\text { Tejido mesenquimal con epitelio } \\
\text { odontogénico } \\
\text { Epitelio en cordones y } \\
\text { yemas con células similares a } \\
\text { odontoblastos }^{17,18}\end{array}$ & $\begin{array}{l}\text { Sin predilección por sexo } \\
\text { Primera-segunda década } \\
\text { Relación premolar y molar } \\
\text { inferior }^{17,18}\end{array}$ \\
\hline Osteoma osteoide & $\begin{array}{l}\text { Bien delimitado, borde } \\
\text { esclerótico } \\
\text { Ovalado, conformación en nido, } \\
\text { con centro mineralizado por } \\
\text { neoformación ósea } \\
\text { Localizado, autolimitante, no }_{\text {invasivo }^{19}}\end{array}$ & $\begin{array}{l}\text { Neoformación ósea con centro } \\
\text { vascular de tejido osteoide } \\
\text { rodeada por hueso compacto }{ }^{19}\end{array}$ & $\begin{array}{l}\text { Predilección sexo masculino } \\
\text { Mandíbula } \\
\text { Dolor patognomónico (cede } \\
\text { con AINEs) }\end{array}$ \\
\hline Cementoblastoma & $\begin{array}{l}\text { Bien delimitado, borde radiolúcido } \\
\text { Radiopaco } \\
\text { En relación con raíz dental, } \\
\text { ausencia de espacio periodontal, } \\
\text { desplazamiento y rizalisis } \\
\text { probables }^{17}\end{array}$ & $\begin{array}{l}\text { Tejido similar a cemento con líneas } \\
\text { de aposición con periferia no } \\
\text { mineralizado (unido a diente) }{ }^{17}\end{array}$ & $\begin{array}{l}\text { Sin predilección por sexo } \\
\text { Segunda-tercera decáda }{ }^{17}\end{array}$ \\
\hline Osteoblastoma & $\begin{array}{l}\text { Bien delimitado, borde radiolúcido } \\
\text { posible, no homogéneo } \\
\text { Radiopaco, mixto } \\
\text { Ausencia de relación directa con } \\
\text { raíz dental }{ }^{21}\end{array}$ & $\begin{array}{l}\text { Hipercelularidad de osteoblastos } \\
\text { y algunas células gigantes tipo } \\
\text { osteoclastos en estroma fibroso }_{\text {vascular }}{ }^{21}\end{array}$ & $\begin{array}{l}\text { Predilección sexo masculino } \\
\text { segunda década } \\
\text { Mandíbula }{ }^{21}\end{array}$ \\
\hline
\end{tabular}




\section{CONCLUSIÓN}

El FCO como patología neoplásica ectomesenquimal sin epitelio odontogénico es poco frecuente, poseyendo predilección por sexo femenino entre la segunda y cuarta décadas de la vida, en zona mandibular posterior.

Para su diagnóstico es necesario conocer las características imagenológicas, las cuales deben ser contrastadas con la histopatología de estudio, descartando un amplio número de diagnósticos diferenciales.

EI FCO posee carácter no agresivo, sin embargo, su crecimiento no es autolimitante, lo que conlleva a ser tratado quirúrgicamente con el fin de evitar alteraciones funcionales y estéticas.

La decisión de tratamiento debe ser argumentada según tamaño, ubicación, relación con estructuras vecinas y actitud del paciente.

La histopatología describe cápsula o pseudocápsula, justificando un tratamiento de enucleación y curetaje que posee un porcentaje de recidiva de $20 \%$, necesitando seguimiento y control por mayor tiempo; la resección marginal presenta menor porcentaje de recidiva, pero pertenece a un tratamiento invasivo, el cual debe ser planificado en reconstrucción y rehabilitación.

El uso de PRF y sticky bone como terapia complementaria en relleno y cicatrización se argumenta en la evidencia asociada al incremento en los procesos fisiológicos mediadores y modulares de remodelación ósea de los maxilares.

\section{REFERENCIAS}

1. Speight PM, Takata T. New tumour entities in the 4th edition of the World Health Organization Classification of Head and Neck tumours: odontogenic and maxillofacial bone tumours. Virchows Arch. 2018; 472 (3): 331-339.

2. Torul D, Kahveci K, Omezli MM, Ayranci F, Erzurumlu UZ. Cemento-ossyfying fibroma of the mandible: report of case. Annals of Dental Specialty. 2019; 7 (1).

3. Domínguez Cuadrado L, Martín-Granizo López R. Análisis clínico, radiológico e histológico de los fibromas cemento-osificantes de los maxilares. Rev Esp Cirug Oral y Maxilofac. 2004; 26 (1): 31-40.

4. Kaur T, Dhawan A, Bhullar RS, Gupta S. Cemento-ossifying fibroma in maxillofacial region: a series of 16 cases. J Maxillofac Oral Surg. 2021; 20 (2): 240-245.
5. Vargas FI, Mayer OC, Hervozo SP, Navia GE. Fibroma cemento osificante: análisis clínico, radiológico e histológico de 2 casos en una misma familia. Int J Odontostomat. 2011; 5 (3): 270-278.

6. Trijolet JP, Parmentier J, Sury F, Goga D, Mejean N, Laure B. Cemento-ossifying fibroma of the mandible. Eur Ann Otorhinolaryngol Head Neck Dis. 2011; 128 (1): 30-33.

7. Ram R, Singhal A, Singhal P. Cemento-ossifying fibroma. Contemp Clin Dent. 2012; 3 (1): 83-85.

8. Gunaseelan R, Anantanarayanan P, Ravindramohan E, Ranganathan K. Large cemento-ossifying fibroma of the maxilla causing proptosis: a case report. Oral Surg Oral Med Oral Pathol Oral Radiol Endod. 2007; 104 (4): e21-e25. doi: 10.1016/j. tripleo.2007.04.026.

9. Hamner JE 3rd, Scofield HH, Cornyn J. Benign fibro-osseous jaw lesions of periodontal membrane origin. An analysis of 249 cases. Cancer. 1968; 22 (4): 861-878.

10. Ahmad M, Gaalaas L. Fibro-osseous and other lesions of bone in the jaws. Radiol Clin North Am. 2018; 56 (1): 91-104.

11. Cecchetti F, Luciani F, Bramanti E, Bartuli FN, Ottria L, Arcuri C. Cemento-ossyfying fibroma juvenile of the oral cavity. Oral Implantol (Rome). 2010; 3 (1): 33-37.

12. Sanchis JM, Peñarrocha M, Balaguer JM, Camacho F. Fibroma cemento-osificante mandibular: Presentación de dos casos y revisión de la literatura. Med Oral Patol Oral Cir Bucal. 2004; 9 (1): 69-73.

13. Eversole LR, Leider AS, Nelson K. Ossifying fibroma: a clinicopathologic study of sixty-four cases. Oral Surg Oral Med Oral Pathol. 1985; 60 (5): 505-511.

14. Davidova LA, Bhattacharyya I, Islam MN, Cohen DM, Fitzpatrick SG. An analysis of clinical and histopathologic features of fibrous dysplasia of the jaws: a series of 40 cases and review of literature. Head Neck Pathol. 2020; 14 (2): 353-361.

15. Mainville GN, Turgeon DP, Kauzman A. Diagnosis and management of benign fibro-osseous lesions of the jaws: a current review for the dental clinician. Oral Dis. 2017; 23 (4): 440-450.

16. Cavalcanti PHP, Nascimento EHL, Pontual MLDA, Pontual ADA, Marcelos PGCL, Perez DEDC et al. Cemento-osseous dysplasias: imaging features based on cone beam computed tomography scans. Braz Dent J. 2018; 29 (1): 99-104.

17. Rajendra Santosh AB, Ogle OE. Odontogenic tumors. Dent Clin North Am. 2020; 64 (1): 121-138.

18. Chrcanovic BR, Brennan PA, Rahimi S, Gomez RS. Ameloblastic fibroma and ameloblastic fibrosarcoma: A systematic review. J Oral Pathol Med. 2018; 47 (4): 315-325.

19. Bhure U, Roos JE, Strobel K. Osteoid osteoma: multimodality imaging with focus on hybrid imaging. Eur J Nucl Med Mol Imaging. 2019; 46 (4): 1019-1036.

20. Ranganath K, Kamath SM, Munoyath SK, Nandini HV. Juvenile psammomatoid ossifying fibroma of maxillary sinus: case report with review of literature. J Maxillofac Oral Surg. 2014; 13 (2): 109-114.

21. Gordon SC, Maclntosh RB, Wesley RK. A review of osteoblastoma and case report of metachronous osteoblastoma and unicystic ameloblastoma. Oral Surg Oral Med Oral Pathol Oral Radiol Endod. 2001; 91 (5): 570-575. 